\section{The case of the missing rule: Memory for reward vs. formal structure in serial-pattern learning by rats}

\author{
STEWART H. HULSE \\ The Johns Hopkins University, Baltimore, Maryland 21218
}

Capaldi and his associates (Capaldi, Blitzer, \& Molina, 1979; Capaldi \& Molina, 1979; Capaldi, Verry, \& Davidson, 1980) report data that, in their view, raise difficulties for an approach to serial-pattern learning in animals based on a cognitive model that presumes that rats encode some representation of a "rule" relating neighboring stimuli (food quantities) in a temporal sequence (Hulse, 1978; Hulse \& Dorsky, 1977, 1979). While my colleagues and I value their concern and attention, there are two difficulties with their analysis that merit comment. First, most of their research does not address fairly the issue of rule learning at all. Second, in spite of their comments to the contrary, significant differences in procedure exist between their research and ours that have important consequences for proper data interpretation. $I$ address these points in turn before suggesting briefly a possible synthesis of the two theoretical approaches.

The literature fails to provide precise, generally accepted definitions of "rules" or "rule learning," and this may be an inherent part of the problem at hand. Briefly, the minimum requirements for a rule lie in a formally defined relationship that systematically maps the elements of one class of events upon the elements from one or more other classes of events. An obvious implication of this definition is that, in practice, a single instance of a formal relationship between two events-say, Stimuli A and B, where $\mathrm{A}>\mathrm{B}-$ neither defines a rule nor, therefore, permits rule learning. It is only when many instances of a class of stimuli, $A_{1} \ldots A_{n}$, are formally related in the same way with instances from another class of stimuli, $B_{1} \ldots B_{n}$, that rule learning may occur. Thus, for example, it is only as a subject learns a series of discrimination problems using different stimuli sharing a common formal relationship that we invoke the idea of a learning set. Similarly, in concept formation, we observe rule learning only as a subject induces a systematic formal relationship that generalizes across many exemplars of the concept. Under simpler circumstances, which involve single Stimuli A and B alone, we generally find it parsimonious to hypothesize stimulus-stimulus or pairedassociate learning.

In bringing models of human serial-pattern learning into the animal laboratory, I have been careful to retain the essence of the cognitively oriented, formally defined rule structures upon which they are based (e.g., Jones, 1974; Restle, 1970). Like all cases of rule learning, these structures typically involve many exemplars of stimulus relationships, which are organized such that a subject can induce some systematic formal principle from the pattern. For example, in long patterns of numbers, such as 1234567 , each number is related to its predecessor such that there are many examples of the NEXT rule, which operates on the ordered set of numbers to generate successive elements of the pattern. People would have little trouble inducing that formal principle, given some brief experience with the pattern, and most would also be able to generalize the rule and supply the next number in the sequence. However, what if we were to reduce exemplars by shortening the pattern? What is the general formal principle underlying the two-element sequence 12 , for example? Is it a NEXT rule on the ordered set of numbers (which would call for a 3 if the pattern were to be extended) or is it an ALTERNATE rule (which would, similarly, call for a 1)? In all cases, the sequence contains insufficient information to make the distinction, and therefore, we have no true rule-based pattern at all. No doubt, people would have little difficulty learning that 2 follows 1 -in the sense of a paired associate-but the two numbers fail to unambiguously define a formal rule that could be accurately generalized to produce other numbers in a sequence. The general point is that the very definition of a serial pattern-for both people and rats-is intimately tied not only to the formal rules from which the pattern is constructed, but also to the opportunity the pattern provides for formal structure to be observed and thereby induced as rules. And, among other things, that opportunity is intimately related to pattern length, that is, to the number of exemplars of the rule in question.

In formulating alternative accounts of our work, Capaldi and his associates have unwittingly taken matters from the domain of rule learning to the domain of paired-associate learning. They have done so on a number of counts. First and foremost, they have simplified their patterns-chiefly by making them shorter-such that rule structures either do not exist or are so impoverished that they no longer fairly and clearly represent serial patterns at all. Second, they have given their subjects relatively little practice and experience with the relevant stimuli when patterns do properly exist. Third, they have sometimes used very long, nonoptimal intervals between successive elements within a pattern, between successive pattern repetitions, or both. Sometimes they have simultaneously distorted a rule-learning approach on two or more of these counts. 
Thus, for Capaldi, Verry, and Davidson (1980), most of the "patterns" are simple pairs of two food quantities. In some instances, two exemplars of a two-element decreasing rule are used (e.g., 4-0 and 16-4), but the pairs are presented 30-min apart and in different orders from one day to the next. Only one case, the 16-4-0 pattern of Experiment 1, incorporates two instances of the same "less than" rule such that a true pattern is formed under optimal temporal conditions. In all cases, including the last, however, just one "pattern"' repetition was used each day, and the rats were therefore exposed to just 16 "pattern" repetitions in all. In earlier work, Capaldi, Blitzer, and Molina (1979) and Capaldi and Molina (1979) used longer three- and four-element patterns, but there, the time intervals between successive elements of the patterns were roughly $4 \mathrm{~min}-$ on a priori grounds, hardly conducive for either multielement associative or rule learning. Again, only 22 pattern repetitions were used, one per day.

In our work, on the other hand, we have consistently used five-element patterns. Small increases in absolute pattern length (from three or four to five elements) may seem inconsequential, but that is not necessarily so, given that the number of direct and remote forward paired relationships in a serial sequence is $[N(N-1) / 2]$, where $N$ is the number of elements in the sequence. Furthermore, we have typically repeated patterns at least four times per test day, using 60 to 100 or so repetitions in an experiment, and we have consistently incorporated brief 15-20-sec intervals between pattern elements. In one instance thus far in which we used shorter patterns to teach a "less than"' rule (Hulse \& Dorsky, 1979), we employed structures that varied from two to four elements in length. But the element sizes also varied substantially from instance to instance, and each pattern occurred in a context that included 15 or so other pattern exemplars. In other words, we were trying to assure that the only consistent feature of our patterns was the rule in question. Perhaps those procedures produced analytic difficulties for Capaldi et al., but the technique was at the very heart of the phenomena we had predicted and were seeking. (Incidentally, Capaldi et al. chose to ignore one of the more interesting results of that experiment-the potent negative transfer generated by the shift from monotonic to nonmonotonic conditions.)

Of course, there is no fault to find with the methods Capaldi and his associates have used, as such, or, of course, with their empirical observations. My objection is with their judgment concerning the domain to which their results apply. In a misdirected effort to achieve parsimony, they have so oversimplified the expression of the rule-learning problem as to destroy its very definition and meaning. However, taken together, their research and ours suggests an idea that may help to identify and characterize the boundaries between learning situations conducive to reward memories and S-S or S-R associative learning, and those conducive to information processing and rule learning. To the extent that patterns are short, contain few exemplars of a potential formal relationship among stimuli, and assure sharp discriminability among pattern elements, associative principles based on reinforcement memories may suffice. However, when information load is increased by using, for example, long patterns with many different stimulus elements, then rats may search for other, consistent within-pattern relationships-such as rules-which, if they exist, could simplify anticipation of pattern elements. Experiments designed to test this general idea are not hard to imagine.

\section{REFERENCES}

Capaldi, E. J., Blitzer, R. A., \& Molina, P. Serial anticipation pattern learning in two-element and three-element series. Bulletin of the Psychonomic Society, 1979, 14, 22-24.

Capaldi, E. J., \& Molina, P. Element discriminability as a determinant of serial-pattern learning. Animal Learning \& Behavior, 1979, 7, 318-322.

Capaldi, E. J., Verry, D. R., \& Davidson, T. L. Memory, serial anticipation pattern learning, and transfer in rats. Animal Learning \& Behavior, 1980, 8, 575-585.

Hulse, S. H. Cognitive structure and serial pattern learning by rats. In S. H. Hulse, H. Fowler, \& W. K. Honig (Eds.), Cognitive processes in animal behavior. Hillsdale, N.J: Erlbaum, 1978.

Hulse, S. H., \& Donsky, N. P. Structural complexity as a determinant of serial pattern learning. Learning and Motivation, 1977, 8, 488-505.

Hulse, S. H., \& Dorsky, N. P. Serial pattern learning by rats: Transfer of a formally defined stimulus relationship and the significance of nonreinforcement. Animal Learning \& Behavior, 1979, 7, 211-220.

Jones, M. R. Cognitive representations of serial patterns. In B. Kantowitz (Ed.), Human information processing: Tutorials in performance and cognition. Hillsdale, N.J: Erlbaum, 1974.

RESTLE, F. Theory of serial pattern learning: Structural trees. Psychological Review, 1970, 77, 481-495.

(Received for publication June 3, 1980; accepted June 30, 1980.) 